\title{
La especialización en Trabajo Social Forense: urgencias desde la intervención
}

\author{
Osvaldo Agustín Marcón \\ Universidad Nacional del Litoral e Universidad Adventista del \\ Plata (Argentina)
}

\section{La especialización en Trabajo Social Forense: urgencias desde la intervención}

Resumen: El artículo propone atender la necesidad de especialización de la intervención en ámbitos judiciales y forenses. Resulta de entrevistar a distintos actores, profesionales, jueces y otros, de la Justicia de Menores de Santa Fe, Argentina, proyectando conceptos teóricos y referencias empíricas más allá de dicho escenario. El enfoque metodológico es cualitativo, y su objetivo central se orienta a explorar las relaciones entre los postuladores legales y los supuestos culturales operantes. El corpus arraiga en el plano filosófico y científico-social para adentrarse en el plano más operativo-instrumental que, finalmente, se condensa en una mirada prospectiva sobre el tema.

Palabras claves: Forense. Especialidad. Intervención. Justicia. Academia.

\section{Especialização em Serviço Social forense: intervenção de emergência}

Resumo: O artigo tem como objetivo abordar a necessidade de especialização da intervenção judicial e forense. É resultado de entrevistas com profissionais, juízes, dentre outros, da Justiça de Menores de Santa Fé, Argentina, projetando conceitos teóricos e referenciais empíricos para além de tais cenários. A abordagem metodológica é qualitativa e seu principal objetivo é investigar as relações entre postulados legais e pressupostos culturais operantes. Finalmente, apresenta um olhar prospectivo sobre o tema.

Palavras-chave: Forense. Especialidade. Intervenção. Justiça. Academia.

\section{The Specialization of Forensic Social Work: Emergencies of intervention}

Abstract: This article proposes serving the need for specialization in interventions in legal and forensic realms. It is the result of interviews with various actors, social workers, judges and others, from the Youth Court of Santa Fe, Argentina, and outlines theoretical concepts and empirical references beyond that situation. The methodological focus is qualitative, and its central objective is to guide an exploration of relations between legal postulates and operating cultural suppositions. The article is rooted in the philosophical and social-scientific planes, to raise more operative-instrumental considerations, which finally are condensed in a prospective look at the issue.

Keywords: Forensic. Specialty. Intervention. Justice. Academy. 


\section{La cuestión a discutir}

Iniciaremos este trabajo incurriendo en una contradicción si tenemos en cuenta lo que luego se pondrá a consideración. Ella consiste en comenzar a describir la cuestión a discutir desde el punto de partida de los Derechos Humanos del Niño que han sido positivizados. Esto, decimos, contradice lo que sigue pues podrá leerse que se piensa a dichos instrumentos en carácter de elementos del sistema convencional, mucho más rico en tanto matriz de pensamiento y acción que como mero sistema de normas jurídicas. Dicho en otros términos, inscribiremos este trabajo en la línea de la sociojuridicidad (KRMPOTIC, 2013) y la sociojudicialidad (MARCÓN, 2016) antes que en los de las binarias juridicidad y judicialidad. Esto equivale a ubicar las normas jurídicas como parte necesaria del ordenamiento social pero no como substancialidad en sí mismo sino como funciones de otros decisivos planos: ideológico, político, cultural y económico. Aceptamos tal ruido argumental por considerar que facilita notoriamente la construcción de la comunicación y, en definitiva, queda relativizado al ser puesto en evidencia.

Otra precisión inicial necesaria es la referencia al tipo de situaciones de conflicto desde las cuales escribimos este artículo. Se trata de las situaciones de conflicto penal, aún cuando muchos de los elementos pueden ser fácilmente extrapolables a otras. Y, entonces, están especialmente presentes los saberes y prácticas desarrollados en torno a los sistemas de responsabilización penal juvenil, fuertemente vigentes en unos países latinoamericanos, especialmente deteriorados en otros, aún en estado germinal en algunos y firmemente cuestionados en la mayoría.

Adentrándonos en nuestro tema, entonces recordemos que toda intervención socio-judicial en el campo infanto-juvenil debe configurarse, indefectiblemente, respetando principios específicamente consagrados en los planos nacional e internacional. Entre ellos tiene una presencia privilegiada el denominado Principio de Especialidad ${ }^{1}$. Su fuerte entidad le viene de los imperativos acordados en la Convención Internacional de los Derechos del Niño (en adelante CIDN) pero también de otros instrumentos centrales entre los cuales se cuentan la conocidas como Reglas de Beijing (o Reglas Mínimas de Naciones Unidas para la Administración de la Justicia de Menores) y la Observación General n. 10 del Comité de los Derechos del Niño.

La necesidad de Especialidad funciona como axioma para la organización de los sistemas de administración de justicia, exigiendo entonces especiales cualidades de las organizaciones de la misma y a sus operadores particulares: básicamente a jueces, fiscales y defensores. Antes de seguir adelante dejaremos dicho, entonces, que formando parte del sistema convencional de derechos de los que gozan los ciudadanos menores de edad, la especialidad es, ante todo, un derecho del que ellos gozan. Esto importa un grado de exigencia superior pues el centramiento en el niño o niña inviste a los mismos de otra naturaleza. Así, la adaptación de los procedimientos, la capacitación específica de los operadores y la diferenciación de las medidas que se adoptan, en relación con las medidas ordinarias para ciudadanos mayores de edad, queda instituida como una exigencia supranacional ineludible. La exigencia se enriquece y complejiza especialmente cuando advertimos que esto incluye el desarrollo de matrices de pensamiento y acción efectivamente especializadas.

Ahora bien: la presencia de Trabajo Social en estos espacios cuenta en la mayoría de los países latinoamericanos con varias décadas de antigüedad. Inclusive, en muchos casos, esa presencia es previa a la CIDN, año 1989, y los demás instrumentos internacionales. Es por ello que esas matrices previas no deben ser descartadas lisa y llanamente pues caeríamos en nuevas expresiones de los modos etapistas de pensar las transformaciones según el cual el año 1989 marcaría un quiebre en la historia. Es cierto que la preocupación por la especialización tiene peso específico luego de dicho hito, situación que establece un rasgo diferencial respecto de las matrices vigentes con antelación. Pero gran parte de su fortaleza viene también de las situaciones historiográficas de cada país. El nacimiento de la CIDN marca transformaciones, con rupturas y continuidades, que impone discusiones y sugiere otras. No obstante esas tensiones son impulsadas para dejar atrás la doctrina de la situación irregular y desarrollar la doctrina de la protección integral. Con ello, sabemos, se intenta sustituir la vieja ideología compasivo-represiva para hacer lugar a la perspectiva de derechos. Pero existen diversas experiencias de protección de derechos en América Latina que son previas a este momento, por lo que no deben ser descartadas de plano, a manos de lo simplemente novedoso. Al interior de lo novedoso no es difícil hallar encriptados distintos componentes que más se inscriben en la vieja lógica que en las renovadas concepciones, como veremos más adelante, razón por la cual no caben ni la negación lisa y llana de la historia ni la fascinación acrítica ante supuestas innovaciones.

No nos explayaremos aquí (las damos por ampliamente conocidas) en las diferencias particulares entre una mirada y otra, es decir entre la Doctrina de la Situación Irregular y la Doctrina de la Protección Integral. Pero nos centramos en algunas cuestiones que impactan sobre Trabajo Social, ahora Forense, con énfasis por identificar algunos puntos que pueden servir para avanzar en el desarrollo del mismo hacia la especificad referida. Pensar en dicha reorganización de la disciplina en función de este Principio de Especialidad exige tener presente lo que decíamos, es decir valorar las virtudes pero también los defectos que la profesión trae 
desde aquella mirada pre-convencional. Solo así, es decir con la historia en el campo de la conciencia profesional, es posible realizar aportes de alto compromiso con la vigencia integral de los Derechos Humanos del Niño.

\section{Algunos de las ventajas en el campo}

Aunque siempre de manera escueta, dejaremos dicho que fue el sistema pre-convencional el que posibilitó la participación interdisciplinaria ${ }^{2}$ en los sistemas de justicia infanto-juvenil (ẢLVAREZ, 2016). Admitir esto es especialmente importante como manera de abrir efectivamente la problematización del campo sin caer, decíamos, en la ensoñación de la mera innovación. Por el contrario, son los sistemas pos-convencionales, especialmente en el campo de la justicia penal juvenil, los que tienden a excluir la visión interdisciplinaria para ubicarla, más que nunca, en el lugar de esos saberes auxiliares a los que se utiliza para dotar de brillo a las intervenciones que, no obstante, son linealmente jurídicas, por tanto monistas en el sentido que a esta expresión le diera el jurista Arnoldo Siperman (2008). En medio de esta presencia interdisciplinaria, y aunque siempre en posiciones subalternas, Trabajo Social tuvo una participación importante. Esto implica todo lo positivo que podemos deducir pero también todas las responsabilidades en la participación de la ya citada compasión-represiva, tan característica de los sistemas tutelares. Esa historia pudo ser capitalizada mucho más en términos de producción teórica y cualificación de la intervención profesional pero, en el grado en que lo fue, también constituye un capital a tener presente.

Toda vez que, ya por estos años, distintos autores proponen pensar desde la complejidad (MORÍN, 2004) y, con ello, desamurallar (NAJMANOVICH, 2008) las disciplinas, bien podemos tomar la palabra y decir que desde Trabajo Social, más aún en el espacio Forense, mucho tenemos para decir. Esta no es una profesión que se haya caracterizado por la construcción de una vez y para siempre de su sujeto (u objeto) de conocimiento y acción. Por el contrario, se trata de una disciplina-profesión (no discutiremos aquí esta cuestión) caracterizada por su habituación a la extranjeridad en tanto rasgo constitutivo de sí misma. Sus fronteras, lejos de estar caracterizadas por la impermeabilidad, siempre tuvieron el rasgo de lo abierto, con todo lo doloroso pero también con toda la maleable robustez que esa suerte de hibridez trae consigo. Es, quizás, una importante ventaja ante otros saberes, fuertemente constituidos en sí y para sí mismos. En esta línea, la idea de especificidad disciplinar ha sido uno de los ejes en la construcción de los saberes tradicionales con los que usualmente TSF interactúa. Los profesionales del campo de las Ciencias Jurídicas, fundamentalmente abogados, no se preguntan usualmente por su rol. Tampoco lo hacen los médicos o los psicólogos. Dan por hecho que ello está suficientemente respondido y, entonces, avanzan. Aquella idea de complejidad que día a día se impone, desde el orden de lo real pero también desde los dispositivos teóricos y jurídicos, interpela fuertemente a esas especificidades que día a día, y aún con diversas resistencias, deben admitir ser fecundadas por saberes externos. Esto, reiteramos, no es extraño a Trabajo Social en general por lo que, para el caso de TSF, constituye un inter-juego de relaciones para el cual pareciera estar especialmente preparado.

Hemos dicho que solo mencionaremos algunas de las ventajas que operan a título de capital para jugar el juego (HELER, 2005) que la participación en este campo (BOURDIEU, 1993) socio-judicial impone. Antes de cerrar, y aunque podríamos extendernos mucho más pues existen muchas otras, mencionaremos una última potencia de TSF que, sabemos, le viene de su formación general pero debe ser aquí reconfigurada. No todas las profesiones están habituadas en términos de habilitación del sujeto con el cual trabajan. En el campo sociojudicial, por ejemplo, el sistema convencional ha introducido la idea del niño como sujeto de derechos con la pretensión de diferenciarlo del otrora pasivo objeto de derechos. Éste último era, según distintas conceptualizaciones, destinatario pasivo de acciones adultocéntricas (BAEZA CORREA, 2010) que, decimos aquí, usualmente encubrían idearios etnocéntricos pues esos adultos pertenecían a las clases sociales centrales, y ejercían su poder sobre niños y familias de clases sociales periféricas. Sin embargo el saber jurídico y las prácticas judiciales tienden a sustituir dicho tipo de relación por otra técnicamente unilateralizada, que parece escuchar al niño tal como la CIDN exige pero, simultáneamente, le quita protagonismo efectivo. Esta merma se produce, básicamente, a partir de la muy común confusión en los escenarios socio-judiciales entre la comunicación y el simple hablar-oír. Así, la vieja tutela compasivo-represiva hizo lugar a la nueva tutela tecnocrática (MARCÓN, 2013). En un caso y en el otro la posibilidad de habilitación de los sujetos decae a manos de la efectiva tendencia a su inhabilitación progresiva. Por ejemplo, en los sistemas penales y ante situaciones concretas, los adolescentes deben decir lo que el defensor indica (por ejemplo negar responsabilidad en hechos de los que se saben responsables ${ }^{3}$ ). Esto, que jurídicamente aparece correcto, contradice diversos planos impuestos desde la CIDN. La voz del niño como parte de una situación integral es, así, tenocráticamente negada en nombre de unas pocas garantías, importantes pero insuficientes. Los protagonistas reales son, cada vez más, los operadores específicos (jueces, fiscales, defensores, profesionales). 


\section{Tensiones a reconfigurar}

Reiteramos que, así como no pretendemos ofrecer un vademécum de potencialidades en el punto anterior, tampoco intentamos poner a consideración un listado de nudos a desatar. Esto no es posible en tanto listado pero tampoco como supuestas limitaciones a superar de una vez y para siempre. Y sí, en cambio, se intenta aportar a ampliar el campo de la conciencia respecto de las tensiones que existen. Gran parte de la tarea que se viene realizando en TSF y, por ende, de los programas de trabajo a futuro, deben romper una diversidad de sesgos deterministas ante la fuerza de los poderes típicos de este campo. Desnaturalizar su presencia en el campo, identificar sus componentes usualmente poco democráticos y desnudarlos en tanto contradicciones constituye parte de la tarea profesional trans-cotidiana. Y entonces, tratándose de contradicciones que pueden reemplazar a otras pero no desaparecer, se trata de empujar hacia su reconfiguración progresiva, en alianza estratégica con otros actores socio-judiciales y socio-jurídicos. En este sentido, muchos especialistas (incluyendo juristas) latinoamericanos ya revisan los reduccionismos penales a los que fuera sometida la CIDN. Dicha crítica refiere a la enorme cantidad de energías volcadas hacia la construcción de sistemas de responsabilidad penal juvenil que debilitaron la rica diversidad originaria de aquel acuerdo internacional. La urgencia por dejar atrás el tutelarismo-represivo y avanzar hacia sistemas garantistas sirvió para que, cual Caballo de Troya, con apariencia externa de compromiso con los DD.HH., la lógica penal ingresara en el sistema convencional. Ya en él, se advierte el desembarco más o menos desembozado de sus armas, con sus distintas variantes ideológicas.

El denominado garantismo, es decir el esfuerzo por desarrollar formas de intervención socio-judicial que respeten las garantías procesales y substanciales penales, es irrenunciable para limitar el avance discrecional de la fuerza estatal-judicial. Ese es un ejemplo de punto de alianza interdisciplinaria. Pero, inexorablemente obligados a pensar desde la CIDN, entendemos que dicho posicionamiento garantista exige mayor creatividad que la copia acrítica de los instrumentos provenientes del sistema penal de mayores, discutibles inclusive en ese

La urgencia por dejar atrás el tutelarismo-represivo $\mathbf{y}$ avanzar hacia sistemas garantistas sirvió para que, cual Caballo de Troya, con apariencia externa de compromiso con los DD.HH., la lógica penal ingresara en el sistema convencional. Ya en él, se advierte el desembarco más

o menos desembozado de sus armas, con sus distintas variantes ideológicas. emplazamiento de la justicia. Cuanto menos se logren estas creaciones más lejos estaremos de respetar, entre otros, el decisivo Principio de Especialidad, vector especificante por excelencia de este campo socio-judicial. Todo esto involucra a Trabajo Social Forense pues la referida estrategia del Caballo de Troya no hace diferencia entre disciplinas profesionales. El compromiso con los Derechos Humanos del Niño puede servir, sabemos esto, para el contrabando de otras intencionalidades.

Hemos dicho que, promoviendo la referida integralidad en la concepción garantista, tanto la CIDN como otros instrumentos internacionales postulan la necesidad de desarrollar sistemas socio-jurídicos especiales para la intervención sobre ciudadanos menores de edad involucrados en situaciones de conflicto penal. Así, impulsan decisiones ejecutivas, legislativas y judiciales que den forma de procedimientos institucionales específicos al compromiso convencional. La presentación de dispositivos institucionales a los que se nomina como especializados pero que reproducen macro y micro operaciones no especializadas, constituye como mínimo un vaciamiento de la CIDN. Por el contrario, la especialidad exige el desarrollo de una racionalidad particular, con consecuencias socio-jurídicas, conceptuales y técnicas determinantes. Por ejemplo, sabemos de la relación dialéctica necesidades-satisfactores: ante la sed, los niños habituados a consumir una famosa gaseosa cola norteamericana piden esa bebida antes que agua. Si el sistema socio-judicial infanto-juvenil ofrece respuestas penales, aunque fueran micro-penales, confirma a los adolescentes en esa condición de actores penales. Y como parte de las mismas operaciones, los niega en su condición de sujetos-adolescentes, privándolos del reconocimiento especial que ese estadio psicoevolutivo requiere. Este es solo un ejemplo de cómo toda vez que los sistemas penales juveniles se dejan subsumir en los sistemas penales ordinarios (de adultos) se viola abiertamente un amplio abanico de garantías internacionalmente consagradas. Allí donde todo esto sucede se abre un abanico de cuestiones ante las cuales siempre algo cabe decir desde la especialidad profesional, para lo cual se requiere formación y actualización tan especializa- 
da como permanente. Subrayemos, de todos modos que esas situaciones ante las cuales siempre se puede y debe decir algo técnicamente eficaz, no son externas a Trabajo Social Forense. No se trata de espectáculos sobre los cuales el profesional debe expedirse. Muy por el contrario, importan lógicas que simultánea y cotidianamente interpelan a la profesión, exigiéndole respuestas mucho más complejas que las usuales.

En este sentido, es indispensable el posicionamiento profesional desde matrices que hagan presente los planos generales (ideológicos, culturales etc.) pero, especialmente en este campo, es forzoso el desarrollo de entramados teórico-prácticos específicos. La histórica ausencia de la formación desde las academias en este campo, a nivel de grado y postgrado, trae consecuencias a atender con urgencia. La presencia de TSF, hemos dicho, no es reciente. Sin embargo ella no ha sido acompañada académicamente, rasgo histórico cuya génesis sería objeto de otro artículo. Pareciera claro que la presencia profesional de TSF en los espacios socio-judiciales no puede ser resuelta como si se tratara de emplazamientos en las sedes judiciales del Ministerio de Desarrollo Social, o sus homónimos en cada caso, desde los cuales se deben gestionar recursos ante las evidencias de derechos sociales vulnerados. Lo social, aquí, está obviamente presente pero bajo las formas socio-judiciales. Y ello, como también sucede en otros espacios, obliga a comprender la lógica socio-judicial y socio-jurídica para incidir eficazmente. Es indispensable gestar acciones que respondan a esa especificidad. Todo esto remite a preguntas tales como la inherente a los insumos básicos, si vale esta nominación, con los cuales trabajar en los espacios socio-judiciales dado que los satisfactores, en sus dimensiones inmateriales e inmateriales, tal como lo plantea Aquín (1995), no forman parte de la especificidad socio-judicial.

En el desarrollo de saberes específicos, convendría identificar rasgos especificantes. En este sentido hagamos presente el hecho de que los socio-judiciales no son dispositivos dadores de bienes que corresponden por derecho sino que, más bien, operan como una instituciones de quita en pro de garantizar reconfiguraciones situacionales. Éste constituye un sesgo central ante el cual TSF debe revisar buena parte de sus supuestos tradicionales. Ante ello cabe la pregunta ¿de qué se dispone entonces para explotar el intersticio representado en la situación socio-judicial? He ahí, quizás, una cuestión central en la especialización profesional.

Para intentar una respuesta conviene preguntar previamente qué es lo que se pretende re-constituir para, luego, identificar qué insumos son necesarios en un contexto obligatorio y de quita. Está a la vista que si el objetivo es, por ejemplo, que el joven retome su escolaridad, será la dependencia estatal específica, usualmente Ministerio de Educación, la encargada de garantizarlo, en el marco de la Doctrina de la Protección Integral. Excepcionalmente, se requerirá de una orden judicial pero si esta es la tarea de TSF, ella aparece altamente asociada a funciones administrativas, y quizás un tanto escuálida. Sin embargo cabe seguir preguntando: en las situaciones de conflicto penal ¿qué significa el hecho penal para los actores, por acción o por omisión, de tal situación? ¿Qué, de lo social, está a la vista y qué debe ser investigado-intervenido socio-judicialmente? ¿Qué debe ser derivado a los ámbitos de las políticas sociales? Desde Matza (2008) sabemos que quien comete un hecho delictivo siempre cuenta, estrictamente, con una razón para ello. Entonces ¿quiénes están involucrados en esa racionalización? ¿Qué personas e instituciones? ¿Cuáles son las representaciones sociales operantes en todos los involucrados? Dejemos propuesto que en cada situación la respuesta a esas preguntas será diferente o al menos exhibirá un matiz particular. Pero en todas aparecerá como común denominador el conflicto de todas las partes para con el orden de lo real. Unos operan ante el conflicto desde lugares hegemónicos y otros desde lugares subalternos, pero todos están en situación de conflicto en relación con la ley. Pero ¿de qué ley hablamos? ¿De una o de una multiplicidad? No nos referimos solamente a la que proporciona el encuadre obligatorio, la norma socio-jurídica, sino todas las leyes que regulan el orden de lo real condicionando a la socio-jurídica pero que, para colmo de complejidad, no son universales.

Todo esto adquiere sentido si coincidimos en que el orden social no se compone substantivamente de lo material sino que se apoya en instituciones imaginarias (CASTORIADIS, 2007), es decir en concepciones socialmente desarrolladas que permiten que los aspectos materiales ocupen tal o cual lugar en un sistema determinado. El proceso transformador puede comenzar por ambas dimensiones, es decir tanto por la material como por la simbólica. Pero para que exista transformación profunda debe, inexorablemente, transformarse el orden simbólico. De allí la relevancia de las intervenciones socio-judiciales y en particular las de TSF. Entones, a efectos de no favorecer supuestos de simple rehabilitación ${ }^{4}$ funcionalista por desvío en relación a la socialización ideal, debería explicitarse contra qué se produjo la rebelión que visibiliza la situación de conflicto. Es evidente que la rebelión es contra la ley pero, y aquí tenemos otra de las claves, es la ley en tanto imperativo cultural. Solo algunas partes de ese imperativo adquirieron, ya en la Modernidad, forma específica de ley positivizada que es encarnada por el orden socio-judicial. ¿Y cuál es ese insumo al que venimos aludiendo para trabajar desde TSF? Precisamente la autoridad que, con sus más y sus menos, se aloja en ese orden socio-judicial. No se trata de la autoridad en sentido de posibilidad de disciplinamiento, como es común que sea entendida y practicada. Se trata, en cambio, de promover la autoridad que adquiere legitimidad ante el joven, en la situación concreta, a partir de introducir elementos de justicia distributiva de responsabilidades. En el diseño de esa co- 
responsabilización aparece una de las claves para la intervención de TSF. Es por esto que la especialidad debe ser fortalecida en tanto garantía de complejidad. Toda vez que las intervenciones son fagocitadas por el binarismo propio de la lógica penal, omitiendo el amplio abanico de componentes que configuran la situación, más lejos estamos de pensar desde una perspectiva compleja, propia de esta especificidad. En este marco, y en tanto proceso constructivo, TSF debería apelar mucho más a la instalación de auténticas formas de comunicación que importen más una racionalidad (HABERMAS, 1987) antes que escenarios de parlantes solazados en la escucha de sus propios argumentos. Recordemos que "cuando la comunicación se basa en el gesto completo estoy comunicando algo que significa cosas diferentes para mí y para el que lo recibe" (DI CARLO, 2008, p. 38). Este gesto completo remite con fuerza a considerar al lenguaje como un medio con decisiva función performativa (AUSTIN, 1982), es decir con capacidad de transformación de la realidad. Éste es un ingrediente substancial para el desarrollo de la especialidad. Es allí donde se abren múltiples posibilidades para, aún en el contexto institucional de quita, instituir espacios restaurativos.

La Especialidad, entonces, obliga a TSF al menos desde dos planos. Uno de naturaleza convencional, es decir impuesto desde el plano de los Derechos Humanos, y el otro de naturaleza ético-política, es decir impuesto a la profesión si la decisión es llevar adelante intervenciones profesionales con impacto efectivo. No se trata, claro está, de subsumir los saberes específicos en los saberes jurídicos, de los abogados, pero sí de incorporar cierta masa crítica de elementos de la disciplina hegemónica para que ella funcione de manera estratégica en las intervenciones profesionales. De otro modo será muy difícil superar aquella afirmación de uno de los reconocidos teóricos del viejo Derecho de Menores en Argentina, Daniel D’Antonio (1999), para quien las Asistentes Sociales son los ojos del juez en el barrio. No se trata, insistimos, de recortar una porción de realidad respecto de la cual desarrollar hiper-especializaciones, sino de inscribir ese recorte en la generalidad, logrando elevados niveles de densidad en la especificidad. Para ello es condición sine qua non admitir que estrategias usualmente útiles en otros espacios dejan de serlo en éste.

Aquí la cuestión de los derechos humanos está obviamente presente, pero con otra dimensión institucional, otros supuestos y otras normativas. Su pasaje al acto adquiere configuraciones distintas a las que toma en, por caso, espacios dependientes de los denominados Poderes Ejecutivos, en sus distintos niveles y expresiones. No se trata de adecuarse acríticamente ni de alienarse tras las bonanzas de espacios dominados por el foucoultiano valor de verdad de las palabras, por delegación jerárquica, sin más. Y sí, en cambio, de reaccionar laboriosa y creativamente para afrontar el desafío que implica re-formarse, luego de advertir que la formación previa era necesaria pero, ya estando en el ámbito de TSF, es indispensable tallar nuevos perfiles. Este esfuerzo es indispensable, so pena de perecer bajo los edificios de la burocracia socio-judicial. Para TSF esto implica afrontar la tarea de elaborar el duelo por el bagaje teórico y operativo instrumental que, tal como está disponible desde la formación académica y desde otros trayectos profesionales, aquí exige profundas revisiones. Este duelo supone desechar parte de -ni más ni menos- toda una identidad profesional-ocupacional que, como se sabe, no es una cuestión accesoria sino inherente a la identidad misma del propio profesional.

Tenemos, entonces, que el Principio de Especialidad exige desde el sistema internacional una urgente transformación a nivel de las matrices de pensamiento-acción en todos los actores judiciales (equipos profesionales, jueces, fiscales, defensores). Es tan urgente desarrollar formas de acusación, defensa y juicio específicamente adaptados a los niños como impulsar modalidades de entrevista, manejo de la información, registro etc., durante las intervenciones de los equipos profesionales. La negación de este plano deja, lisa y llanamente, sin voz a los niños. Pensando la situación desde la intervención, encontramos diversos nodos que requieren trabajos específicos. Así, por ejemplo, el espacio socio-judicial suele tener como característica la confusión indagatoria-entrevista. Suele pensarse que entre una y otra técnica hay simples diferencias de operadores, sin advertir que la lógica de pregunta-respuesta-entrecruzamiento-de-respuestas, propia de la indagación socio-judicial no es la misma que la de la entrevista-situacionalidad-interpretación, propia de la entrevista profesional. Así por ejemplo, la coincidencia-disidencia entre unos y otros relatos suele ser tomada como un análisis de los sentidos latos de las palabras, sin advertir que los planos de indagación de una y de otra son diferentes. Respecto de esta técnica de TSF, la entrevista, podríamos explayarnos mucho más pero, dado que aquí la traemos solo como ejemplo de cuestiones a especializar, limitémonos a agregar que si la Especialidad es por sobre todo un derecho que le asiste al niño judicializado, la entrevista especializada también lo es. Se trata, como sabemos, de un recurso profesional con múltiples aplicaciones en los ambientes socio-judiciales. Aprovechemos para señalar que, como sabemos, el espacio físico integra el conjunto de condiciones necesarias para que la técnica no violente a la persona que recurre a un servicio y debe exponer aspectos íntimos de su vida. Sin embargo, es demasiado común que en los lugares donde se aplica esta técnica coexistan varias personas trabajando, computadoras, puertas que se abren y cierran junto a teléfonos que suenan a la par de un amplio abanico de otras interferencias. La ubicación del lugar físico, su aislamiento sonoro, las restricciones al ingreso o la disponibilidad horaria son requisitos estrictamente básicos para que, entre otras cosas, no se 
configuren situaciones de violencia institucional para con el niño. Esto es así por varias razones pero, fundamentalmente, porque la entrevista es un momento del proceso de intervención socio-judicial en el que se trasciende lo meramente administrativo para tomar contacto con las configuraciones más profundas del conflicto de la situación y de los sujetos involucrados. Y si bien gran parte de esto también sucede en otros espacios estatales, aquí estamos ante situaciones en las cuales la fuerza del Estado, con el monopolio legítimo de uso de la fuerza, y con las fuerzas policiales latinoamericanas como parte del problema (SAÍN, 2008), el escenario adquiere otros contornos.

Antes que requisitos técnicos tales condiciones deben expresar el respeto institucional por la CIDN, en cuyo articulado se encuentra el Derecho a la Intimidad. En estrecha relación aparece otro ejemplo de dimensión a discutir en los espacios de TSF: el secreto profesional. No se trata de un recurso demasiado desarrollado en la profesión, aún cuando está presente la mayoría absoluta de los instrumentos que regulan el ejercicio profesional (MARCÓN, 2008). Entrevistar a un niño, o a su familia, en espacios socio-judicial y técnicamente adecuados está relacionado con todo esto y también con la posibilidad de eficacia en la reconfiguración de situaciones a través del ya referido insumo básico. No se trata solamente de guardar secreto o preservar la intimidad por la obligación que está más a la vista, sino por las más profundas, inherentes al derecho del niño a ser tratado de una manera profesionalmente eficaz.

Podríamos enlistar una multiplicidad de aspectos que violan la especialización y que comprometen la voz de TSF. Éstos van desde los mencionados procedimientos policiales, penitenciarios y del personal de contacto en general, incluyendo el perfil del personal administrativo y de servicio que debe tener especial vinculación en las sedes judiciales, hasta los aspectos edilicios de los dispositivos institucionales. La especialidad, entonces, constituye un horizonte de trabajo particularmente exigente pero, simultáneamente, un proceso de transformación permanente sin el cual el avance hacia un tratamiento adaptado será imposible.

\section{Conclusiones}

La intervención profesional en los espacios socio-judiciales infanto-juveniles se presenta, en principio, como un ámbito particularmente contradictorio para Trabajo Social. Aceptar que la profesión puede respetar sus principios fundamentales en ese contexto, y simultáneamente retroalimentar su legitimidad de base es una de las convicciones que están presentes en este artículo. Los debates inherentes a la necesidad de especialización en Trabajo Social Forense se inscribe, como mínimo, en otro mayor que podemos graficar en la oposición generalistas vs. especialistas. Hemos planteado, en este caso, nuestra posición en favor de la segunda visión aunque no con exclusión de la primera sino postulando su fortalecimiento como conjunto de sentidos para las operaciones específicas.

El desarrollo del profesionalismo, nunca aséptico ni tecnocrático, encuentra especiales desafíos en el ámbito socio-judicial. Éstos se relacionan con la verticalidad en el ejercicio del poder, típica del ámbito puertas adentro y puertas afuera. En ese escenario la disciplina se encuentra subalternizada respecto de otros saberes como el jurídico pero también, en ocasiones, frente a otros saberes subalternos respecto del jurídico (por caso el saber médico o los saberes psicológicos). Desarrollar, entonces, un relato profesional con posibilidades de entablar también en ese plano algún tipo de racionalidad comunicativa, exige a la disciplina subalterna conocer los intersticios de la hegemónica para, así, poder explorar y explotar las grietas que siempre existen. En esta línea, TSF debe agudizar la mirada sobre sí mismo advirtiendo cuántas de sus operaciones cotidianas tienen alguna trascendencia y cuántas de ellas corren por la vía de la burocratización que, sabemos, no es solo una cuestión de grises expedientes (sellos etc.) sino, lo que es más importante, de modos de pensar y actuar. Allí tenemos, como queda dicho, la urgencia por estimular la creatividad. Para ello existen potencialidades, propias de la profesión, que son mucho más robustas en otros ámbitos pues fueron pensadas para ellos (ámbitos más específicos, de desarrollo de políticas sociales en su acepción restringida). Aún así, son capitales pre-existentes.

Desde allí, es decir desde esas potencialidades, entonces, conviene identificar progresivamente los puntos de tensión. Ya no solo de TSF, de manera endogámica, sino en abierta interacción con todos los espacios de saberes que adviertan que la flexibilización de fronteras disciplinares ya no es una opción sino una exigencia en los modos actuales de producción de conocimiento y pasaje a la acción.

\section{Referencias}

ÁLVAREZ, A. Todos los niños tienen derecho al debido proceso. Disponible en: www.cuestionsocial.com.ar. Visitada en 03 mar. 2016. AQUíN, N. Acerca del objeto del Trabajo Social en Acto Social. 1995. Disponible en: http:www.ts.ucr.ac.cr. Visitada en: 2 mar. 2016. 
AUSTIN, J. Cómo hacer cosas con palabras: palabras y acciones. Madrid: Paidos, 1982.

BAEZA CORREA, J. y ot. América Latina: un espacio de rostros juveniles diferentes. Centro de Estudios en Juventud (Ceju). Santiago de Chile: Universidad Católica Silva Henríquez, 2010.

BOURDIEU, P. Génesis y estructura del campo burocrático. Actes de las Recherches en Sciences Sociales, p. 96-97, 1993.

CASTORIADIS, C. Transformación social y creación cultural. In: YAGO, F. Insignificancia y autonomía: debates a partir de Cornelius Castoriadis. Buenos Aires: Biblos, 2007.

D’ANTONIO, D. Derecho de Menores. Buenos Aires: Astrea, 1999.

DI CARLO, E. El método de Trabajo Social profesional. Un aporte a su pedagogía universitaria. Mar del Plata: Grupo EiMets, 2008. HABERMAS, J. Teoría de la acción comunicativa. Madrid: Taurus, 1987.

HELER, M. Ciencia incierta. La producción social del conocimiento. Buenos Aires: Biblos, 2005.

KRMPOTIC, C. Conflicto entre pares en el escenario hospitalario. Aspectos técnicos, legales y éticos en la atención al paciente desde los Servicios Sociales. In: KRMPOTIC C. Culturas Profesionales. Poder y prácticas en Salud. Madrid: Miño y Dávila Editores, 2013. MARCÓN, O. El secreto profesional en Trabajo Social. Buenos Aires: Espacio Editorial, 2008.

. La responsabilización penal juvenil como nuevo relato cultural: ¿Del amor por los niños al odio hacia los menores? Buenos Aires: Espacio Editorial, 2013.

. La justicia infanto-juvenil en la mira: clínica de la intervención en contextos deinequiedad. Buenos Aires: Miño y Dávila, 2016. MATZA, D. Técnicas de neutralización: una teoría de la delincuencia. Salvador: Cuadernos CRH, 2008.

MORÍN, E. La Epistemología de la Complejidad. En: Gazeta de Antropología, n. 20. Disponible en: http:// www.pensamientocomplejo.com.ar. Visitada en: 6 jul. 2008.

NAJMANOVICH, D. Interdisciplina. Artes y riesgos del arte dialógico. 2008. Disponible en: http://www.pensamientocomplejo.com.ar. Visitada en: 2 jun. 2013.

SAÍN, M. El Leviatán Azul: política y policía en la Argentina. Buenos Aires: Siglo XXI, 2008.

SIPERMAN, A. La ley romana y el mundo moderno. Juristas, científicos y una historia de la verdad. Buenos Aires: Biblos, 2008.

\section{Notas}

1 Este Principio implica también el de Especificidad, diferente pero complementario del primero. No desarrollaremos aquí este aspecto pues a los efectos del artículo no es necesario. Pero entiéndase que en este artículo toda referencia al primero hace también referencia al segundo.

2 Usaremos este término sin distinción entre multi, inter, transdisciplina u otras.

3 Nos explayaremos, más adelante, en relación con la noción de responsabilidad que entendemos debe ser pensada en términos de co-responsabilidad.

4 Resocialización, reinserción etc.

\section{Osvaldo Agustín Marcón}

osvaldomarcon@gmail.com

Doctor en Ciencias Sociales en la Universidad Nacional del Litoral

Docente en la Escuela de Servicio Social de la ciudad de Santa Fe

\section{Escuela de Servicio Social de la ciudad de Santa Fe}

Piedrabuena n. 2620

Santa $\mathrm{Fe}$ - Argentina

CP: 3000 